\title{
Cutaneous toxicities of epidermal growth factor receptor inhibitors: A prospective study in 60 Asian patients.
}

\author{
Leena Chularojanamontri, ${ }^{1}$ Papapit Tuchinda, ${ }^{1}$ Chayanee Likitwattananurak, ${ }^{1}$ Kamolwan Pongparit ${ }^{1}$ Chuda Rujitharanawong, ${ }^{1}$ \\ Suthinee Ithimakin, ${ }^{2}$ Kanokvalai Kulthanan ${ }^{1}$
}

\begin{abstract}
Background: Several prospective studies have been conducted in epidermal growth factor receptor (EGFR) inhibitor -related cutaneous reactions in Caucasian patients, but prospective studies in Asian populations are scarce.

Objective: To investigate the cutaneous side effects of EGFR inhibitors in Asian cancer patients and to assess tumor response to dermatologic manifestations.

Methods: Sixty patients with lung or colorectal cancer who were receiving EGFR inhibitors were prospectively followed for at least one year by oncologists and dermatologists.

Results: Of 60 patients (33 males, 27 females), 46 lung cancer patients received erlotinib ( $\mathrm{n}=29)$ and gefitinib ( $\mathrm{n}=17)$. Cetuximab was prescribed in 14 colorectal cancer patients. Fifty-eight patients (58/60, 96.7\%) developed cutaneous reactions. The most common reactions were xerosis $(82.8 \%)$, acne $(79.3 \%)$, and skin desquamation $(62.1 \%)$. Most reactions were mild and well-tolerated. Of 14 patients who had severe reactions, temporary treatment interruption was necessary in 3 patients and a decreasing dose was required in another 3 patients. There were no statistically significant differences in type, severity, or number of cutaneous reactions between responders (29/58) and non-responders (29/58) to EGFR inhibitors. At median follow-up time of $11.92 \pm 1.08$ months, no patient died from cutaneous toxicities. Nine patients died from cancer and 11 patients lost to follow-up.
\end{abstract}

Conclusion: In this Asian population, almost all patients (96.7\%) developed cutaneous toxicities of EGFR inhibitors. Xerosis, acne, and desquamation were common in Asian cancer patients. Most reactions were mild and well tolerated. Due to limited number of patients, this study did not show significant associations between cutaneous toxicities and tumor response.

Keywords: epidermal growth factor receptor inhibitors, cutaneous toxicities, cetuximab, erlotinib, gefitinib

\section{From:}

Department of Dermatology, Faculty of Medicine Siriraj Hospital, Mahidol University, Bangkok, Thailand

Division of Oncology, Department of Medicine, Faculty of Medicine Siriraj Hospital, Mahidol University, Bangkok, Thailand

\section{Introduction}

Human epidermal growth factor receptors (EGFRs), which express in normal human skin tissue, are responsible for cell growth and the proliferation of keratinocytes, and the outer root sheath of hair follicles. ${ }^{1}$ Overexpression of EGFRs may lead to uncontrolled cell growth, proliferation, angiogenesis, and metastasis. Previous studies showed that there is an overexpression of EGFRs in some solid cancers, such as non-small-cell lung cancer (NSCLC), colorectal cancer, and head and neck cancer. ${ }^{1,2}$

\section{Corresponding author:}

Papapit Tuchinda

Department of Dermatology, Faculty of Medicine Siriraj Hospital,

Mahidol University

2 Wanglang Road, Bangkoknoi, Bangkok 10700, Thailand Email: papapitt@gmail.com

As a result of this underlying pathway in tumor proliferation, EGFR inhibitors were developed for the treatment of several chemotherapy-resistant solid cancers such as NSCLC, EGFR -positive colorectal cancer, and squamous cell carcinoma of head and neck. ${ }^{3}$

EGFR inhibitors are categorized into the following two groups: tyrosine kinase inhibitors and chimeric monoclonal antibodies. ${ }^{3,4}$ Tyrosine kinase inhibitors bind to the tyrosine 
kinase portion of EGFRs, which leads to a decrease in cell growth and differentiation of tumor cells. Chimeric monoclonal antibodies compete with EGFR ligands, resulting in down -regulation of transmembrane EGFRs. ${ }^{3-6}$ Unlike conventional cytotoxic chemotherapies, anti-EGFR therapies do not usually cause myelosuppression, neuropathy, or significant nausea, because they have high specificity to EGFR. Common adverse effects of EGFR inhibitors are cutaneous toxicities, which have been reported in $45-100 \%$ of patients. ${ }^{1,4,6}$ Roe, et al. investigated cutaneous side effects in 30 Caucasian patients during treatment with EGFR inhibitors. They found that acneiform eruption, followed by painful fissures in palms and soles and paronychia were the most common cutaneous reactions. ${ }^{1}$ Another study that retrospectively evaluated 99 Asian patients found that the most common cutaneous event was xerosis (52.5\%), followed by acneiform eruption (27.3\%), erythematous maculopapular rash (11.1\%), mucositis (6.7\%), and paronychia (5.1\%). ${ }^{7} \mathrm{~A}$ systematic review and meta-analysis involving 6,798 patients reported that cutaneous eruptions were associated with longer progression-free survival and overall survival. ${ }^{8}$ Several prospective studies have been conducted in EGFR inhibitor-related cutaneous reactions in Caucasian patients, but prospective studies in Asian populations are scarce.

The aim of this study was to investigate the cutaneous side effects of EGFR inhibitors in Asian cancer patients and to assess tumor response to dermatologic manifestations.

\section{Material and Methods}

The protocol for this prospective study was approved by the Siriraj Institutional Review Board (SIRB), Faculty of Medicine Siriraj Hospital, Mahidol University, Bangkok, Thailand. Patients with stage IV cancer who were being started with EGFR inhibitors at the Oncology Unit, Department of Medicine, Siriraj Hospital were enrolled and followed during the 2013 to 2016 study period. All patients were followed by oncologists and dermatologists for at least one year. Demographic data, cancer type with background therapy, duration from drug initiation to the first cutaneous reaction, and therapy for cutaneous toxicities were recorded and analyzed. Cutaneous toxicities were graded as $1,2,3,4$, and 5 according to Common Terminology Criteria for Adverse Events (CTCAE) guideline version 3.0, United States Department of Health and Human Services, National Institutes of Health, National Cancer Institute. ${ }^{59}$ Overall survival, progression-free survival, and tumor response evaluation were assessed using the Response Evaluation Criteria in Solid Tumors (RECIST) guideline. For tumor response evaluation, complete response (CR) was defined as disappearance of all target lesions. Partial response (PR) was defined as reduction of $\geq 30 \%$ in the sum of diameters of target lesions from baseline. Progressive disease (PD) was diagnosed if there was at least a $20 \%$ increase in the sum of diameters of target lesions. Change in the sum of diameters of target lesions that did not meet one of the above-mentioned criteria was defined as stable disease (SD). ${ }^{10}$ In this study, patients who experienced SD, PR, or CR as the best response at some period of time after EGFR inhibitors treatment were defined as responders. Non-responders were defined as patients who met with PD criteria after EGFR inhibitors treatment as the best response. Cutaneous reactions and severity between responders and non-responders were compared. Written informed consent was obtained from all study participants prior to their inclusion in this study.

\section{Statistical analysis}

Data were analyzed using PASW Statistics version 18.0 (SPSS, Inc., Chicago, IL, USA). Descriptive statistics were used to describe demographic data, cancer type, and duration from drug initiation to the first cutaneous reaction. Associations between cutaneous toxicities and tumor response were determined using Chi-square test or Fishers's exact test. A $p$-value less than 0.05 indicated statistical significance.

\section{Results}

A flow diagram of patient enrollment and patient outcome at the end of the study is shown in Figure 1. A total of 60 patients (33 males, 27 females) with a mean age of $66.9 \pm 10.7$ years were included. Demographic data of our patients is shown in Table 1. All patients received EGFR inhibitor as a palliative treatment for metastatic lung or colorectal cancer. At the time of starting EGFR inhibitor, none of eligible patient experienced higher than grade 1 toxicity from previous cancer treatment. Forty-six patients had NSCLC and received tyrosine kinase inhibitors. Of those 46 patients, oral daily erlotinib 150 $\mathrm{mg}$ and oral daily gefitinib $250 \mathrm{mg}$ were prescribed in 29 and 17 patients, respectively. Monoclonal antibodies (cetuximab) with an initial loading dose of $400 \mathrm{mg} / \mathrm{m}^{2}$ was prescribed in 14 patients with colorectal cancer. All patients had stage IV cancer. Fifty-eight of 60 (96.7\%) patients receiving EGFR inhibitor treatment developed cutaneous reactions. Number of responders was equal to that of non-responders. With the median follow up time of $11.92 \pm 1.08$ months, 9 patients died

Table 1. Demographic data of 60 patients treated with epidermal growth factor receptor (EGFR) inhibitors

\begin{tabular}{lc}
\hline Demographic data & N/ 60 patients (\%) \\
\hline Males & $33(55)$ \\
Females & $27(45)$ \\
\hline Underlying diseases & $28(46.7)$ \\
$\quad$ Hypertension & $13(21.7)$ \\
Diabetes mellitus & $12(20)$ \\
Dyslipidemia & \\
Types of cancer & $46(76.7)$ \\
Non-small-cell lung cancer & $14(23.3)$ \\
Colorectal cancer & \\
\hline Previous treatments before EGFR inhibitors therapy & $41(68.3)$ \\
Conventional chemotherapy & $1(1.7)$ \\
Radiotherapy & $6(10)$ \\
Conventional chemotherapies + Radiotherapy & \\
\hline Patients treated with tyrosine kinase inhibitors & $29(48.3)$ \\
$\quad$ Erlotinib & $17(28.3)$ \\
Gefitinib & \\
Patients treated with chimeric monoclonal antibodies & $14(23.3)$ \\
Cetuximab & \\
Concurrent treatments with EGFR inhibitors therapy & $21(35)$ \\
None & $1(1.7)$ \\
Conventional chemotherapy & \\
Radiotherapy & \\
\hline
\end{tabular}




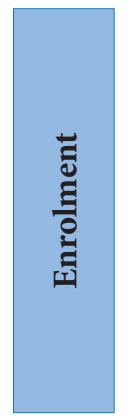

60 Patients with cancer stage IV treated with EGFR inhibitors were enrolled at oncology unit

All patients were followed by oncologists and dermatologists for at least one year

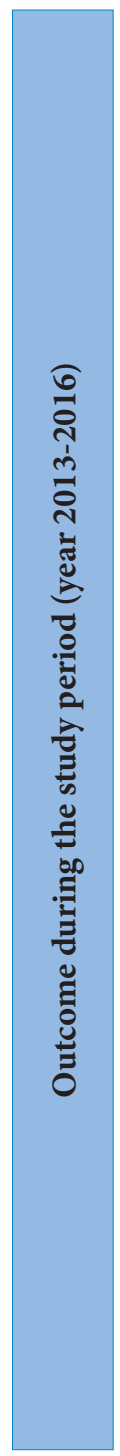

\begin{tabular}{|ccc|}
\hline $\begin{array}{c}\text { Erlotinib } \\
(\mathrm{n}=29)\end{array}$ & $\begin{array}{c}\text { Gefitinib } \\
(\mathrm{n}=17)\end{array}$ & $\begin{array}{c}\text { Cetuximab } \\
(\mathrm{n}=14)\end{array}$ \\
\hline
\end{tabular}
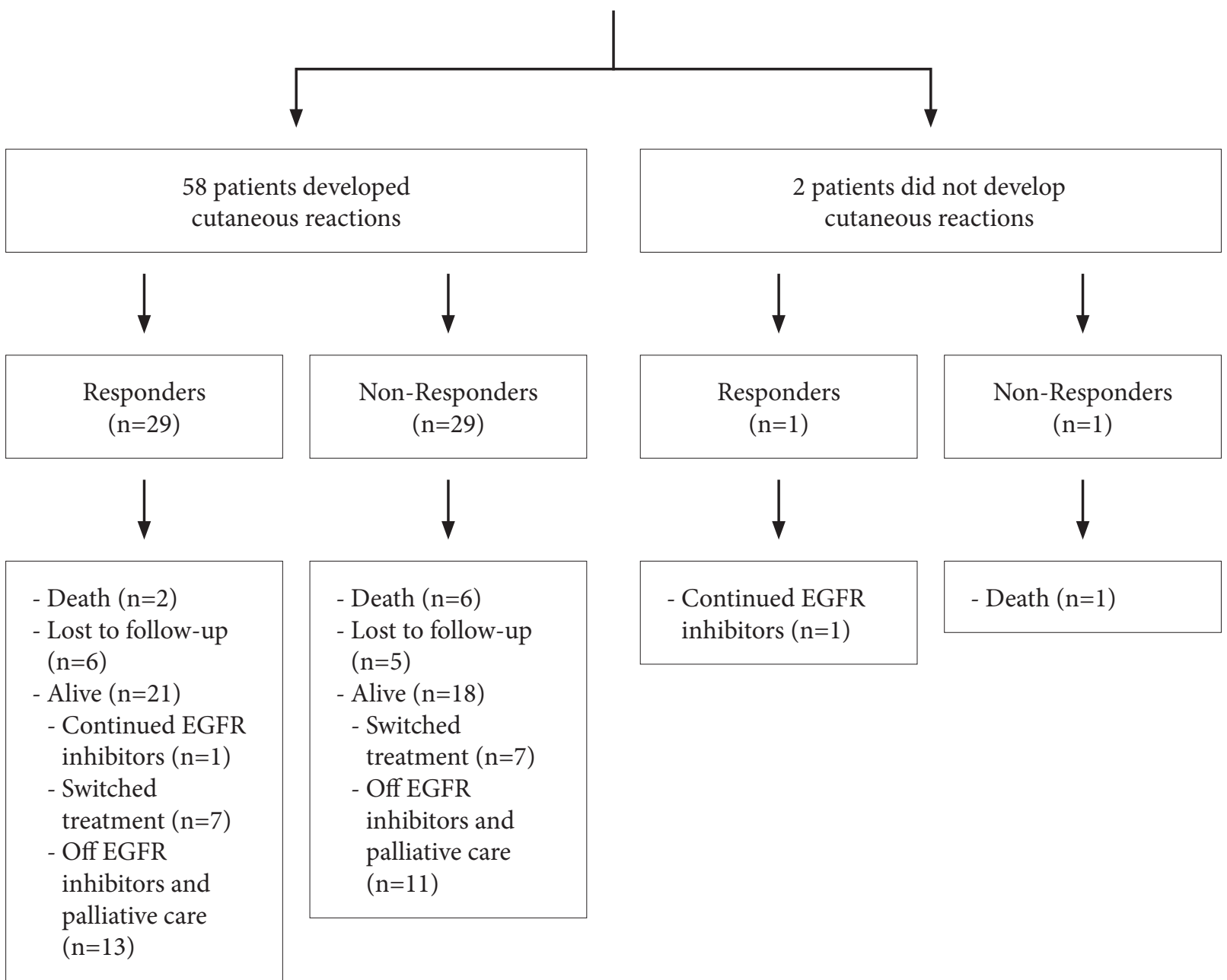

Figure 1. Flow diagram of patient enrollment and outcome of epidermal growth factor receptor (EGFR) inhibitors

from cancer and 11 patients lost to follow-up. Among 40 patients who were alive on the date of data cut-off, EGFR inhibitors were eventually discontinued in 38 patients because of progressive disease. Mean duration of EGFR inhibitor treatment was $8.32 \pm 8.03$ months and $5.83 \pm 4.28$ months in lung cancer and colorectal cancer patients, respectively.

The most common cutaneous toxicities in 58 patients were xerosis (48/58, 82.8\%), acne/papulopustular rash (46/58, 79.3\%), and skin desquamation $(36 / 58,62.1 \%)$. Pruritus without skin xerosis and desquamation, mucositis, and trichomegaly/hypertrichosis were found in only $13.8 \%(8 / 58), 8.6 \%(5 / 58)$, and $5.2 \%(3 / 58)$ of patients, respectively. Figure 2 demonstrates cutaneous toxicities from each EGFR inhibitor. Xerosis was more common in patients taking chimeric monoclonal antibodies (cetuximab) than in patients taking tyrosine kinase inhibitors. There were no statistically significant differences 
in cutaneous toxicities between tyrosine kinase inhibitors and chimeric monoclonal antibodies. Type and time of onset of cutaneous reactions after initiation of EGFR inhibitors is shown in Figure 3. Acne/papulopustular eruption, xerosis, desquamation, and pruritus commonly occurred within one month after starting treatment. Of 46 patients with acne, 54.3\% developed papulopustular lesions within two weeks. The peak duration to the development of nail involvement (paronychia and/or painful fissures) was 6 to 8 weeks. Alopecia and trichomegaly occurred after 10 weeks of treatment.

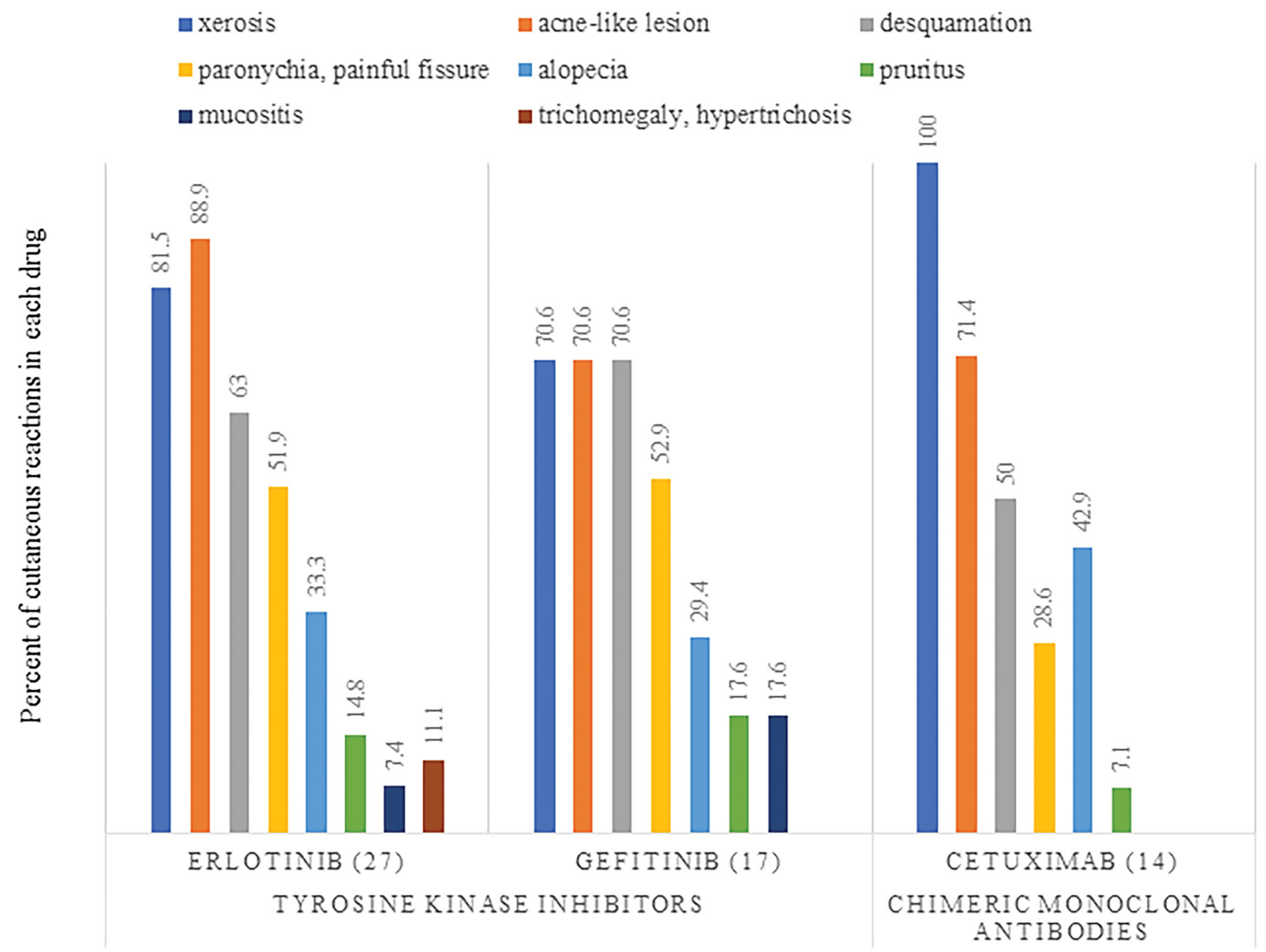

Figure 2. Cutaneous toxicities among types of epidermal growth factor receptor inhibitors

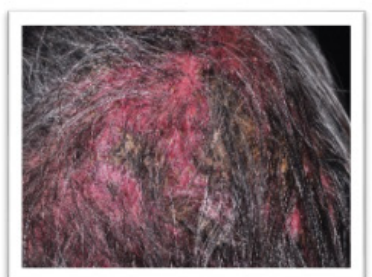

Papulopustular eruption on scalp

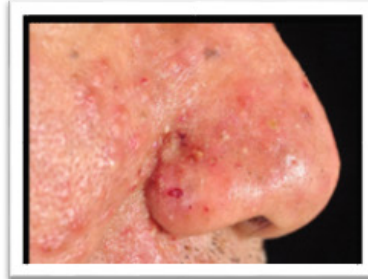

Acne-like lesion

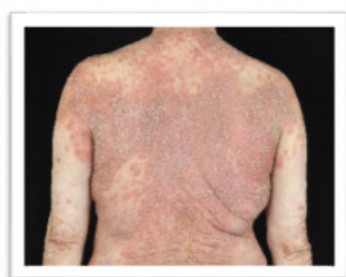

Severe desquamation

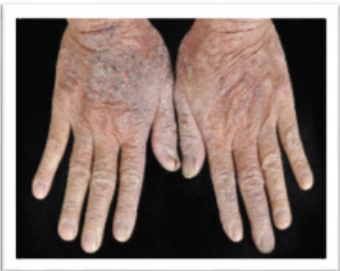

Xerosis and desquamation

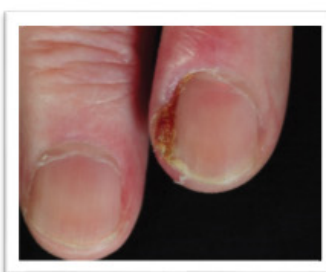

Paronychia

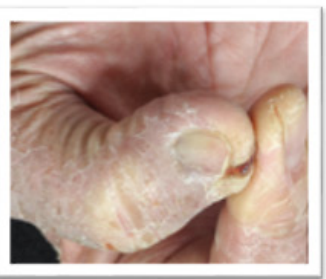

Painful fissure

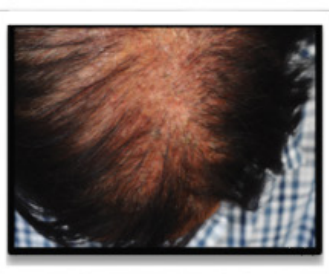

Alopecia

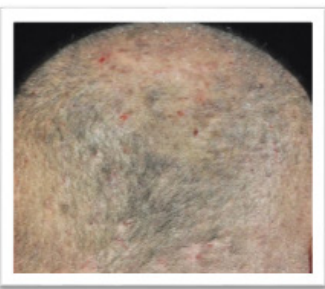

Alopecia

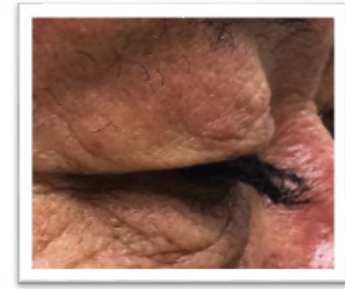

Trichomegaly

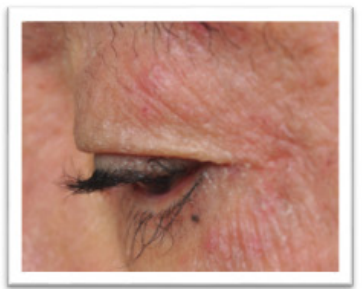

Trichomegaly

Figure 3a. Acne, xerosis, desquamation, and pruritus commonly occurred within one month after treatment. The peak duration of nail involvement (paronychia and/or painful fissures) was 6 to 8 weeks. Alopecia and trichomegaly occurred at week 10 


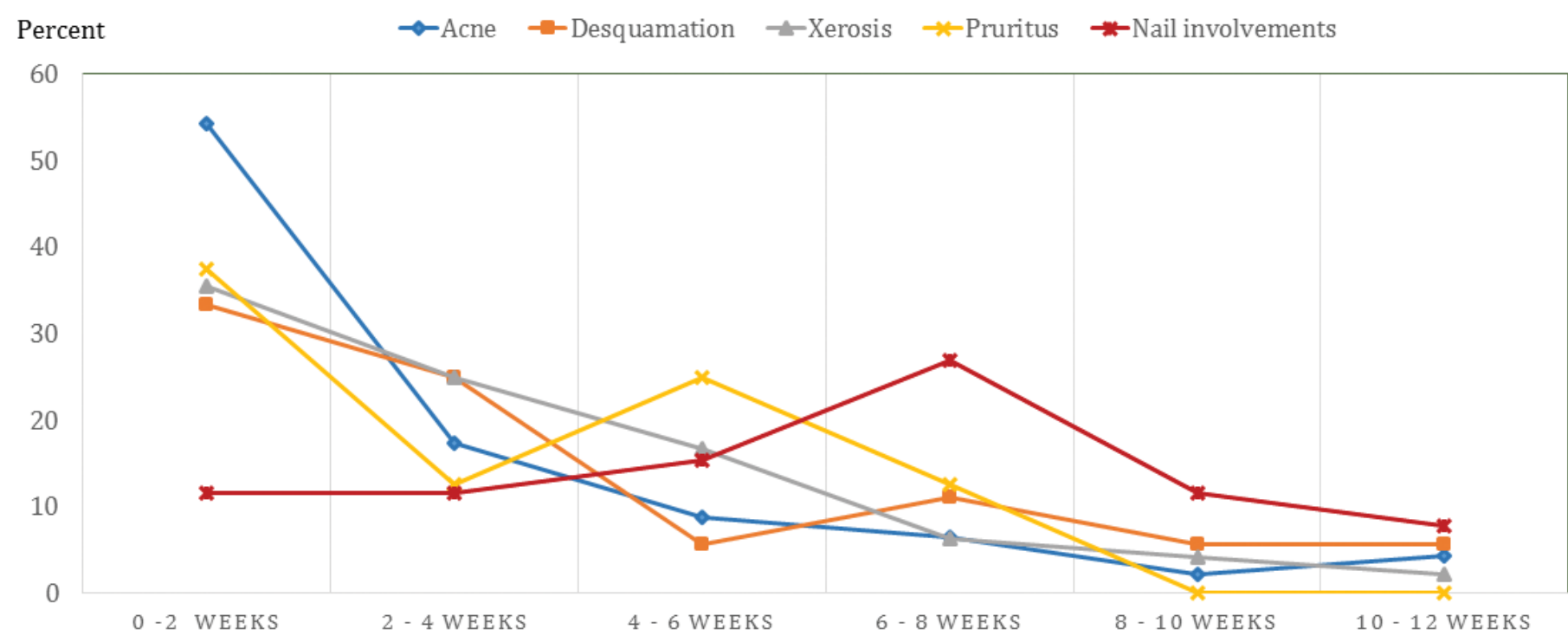

Figure 3b. The frequency of different clinical types of cutaneous reactions associated with duration of anti-EGFR treatment

Table 2. Grading severity of cutaneous reactions to epidermal growth factor receptor inhibitors

\begin{tabular}{lccc}
\hline Cutaneous reactions & Overall & Grades 1-2 & $\geq$ Grade 3* \\
\hline Xerosis, n (\%) & $48 / 58(82.8)$ & $45 / 48(93.7)$ & $3 / 48(6.2)$ \\
\hline Acne-like lesion, n (\%) & $46 / 58(79.3)$ & $41 / 46(89.1)$ & $5 / 46(10.9)$ \\
\hline Desquamation, n (\%) & $36 / 58(62.1)$ & $31 / 36(86.1)$ & $5 / 36(13.9)$ \\
\hline $\begin{array}{l}\text { Paronychia/painful } \\
\text { fissure, n (\%) }\end{array}$ & $27 / 58(46.6)$ & $27 / 27(100)$ & $0 / 27(0)$ \\
\hline Alopecia, n (\%) & $20 / 58(34.5)$ & $20 / 20(100)$ & $0 / 20(0)$ \\
\hline $\begin{array}{l}\text { Pruritus } \\
\text { ** }, \mathrm{n}(\%)\end{array}$ & $8 / 58(13.8)$ & $7 / 8(87.5)$ & $1 / 8(12.5)$ \\
\hline
\end{tabular}

* Grade 3 or higher indicates severe reactions, including those with pain, disfigurement, ulceration, or desquamation involving $>50 \%$ of the body surface area or that interfere with activities of daily living

** Pruritus without skin xerosis and desquamation
Most patients were able to tolerate cutaneous reactions, because the level of severity of most reactions was not severe (Table 2). There was no fatal cutaneous toxicity founded in this cohort. Of 14 patients with $\geq$ grade 3 cutaneous reactions, 3 patients needed temporary interruption of EGFR treatment while another 3 patients required dose reduction. All patients were improved as a result of EGFR inhibitor modification with symptomatic treatment and then were able to resume the same treatment regimen at a later date. With regard to papulopustular reaction, bacterial cultures from pustular lesions from 11 patients showed positive for methicillin -sensitive Staphylococcus aureus in 10 patients (90.9\%) and sterile pustule in 1 patient (9.1\%). Treatment for acne-like lesions included topical antibiotics (clindamycin and erythromycin), $2 \%$ salicylic acid, and topical retinoids. Systemic

Table 3. Cutaneous reactions and severity between responders and non-responders

\begin{tabular}{|c|c|c|c|}
\hline & Responders $(n=29)$ & Non-responders $(n=29)$ & $p$-value \\
\hline \multicolumn{4}{|l|}{ Cutaneous reactions } \\
\hline Acne, n (\%) & $24(82.8 \%)$ & $22(75.9 \%)$ & 0.52 \\
\hline Desquamation, n (\%) & $16(55.2 \%)$ & $20(69.0 \%)$ & 0.28 \\
\hline Xerosis, n (\%) & $23(79.3 \%)$ & $25(86.2 \%)$ & 0.49 \\
\hline Nail, n (\%) & $14(48.3 \%)$ & $13(44.8 \%)$ & 0.79 \\
\hline Alopecia, n (\%) & $13(44.8 \%)$ & $7(24.1 \%)$ & 0.10 \\
\hline \multicolumn{4}{|l|}{ Acne severity } \\
\hline Intervention not indicated, n (\%) & $6(20.7 \%)$ & $6(20.7 \%)$ & 1.0 \\
\hline Intervention indicated, $\mathrm{n}(\%)$ & $15(51.7 \%)$ & $14(48.3 \%)$ & 0.80 \\
\hline Associated with pain, disfigurement, ulceration, and desquamation, n (\%) & $3(10.3 \%)$ & $2(6.9 \%)$ & 0.65 \\
\hline \multicolumn{4}{|l|}{ Xerosis severity } \\
\hline Asymptomatic, n (\%) & $13(44.8 \%)$ & $18(62.1 \%)$ & 0.19 \\
\hline Symptomatic, not interfering with activities of daily living (ADL), n (\%) & $10(34.5 \%)$ & $4(13.8 \%)$ & 0.07 \\
\hline Symptomatic, interfering with activities of daily living (ADL), n (\%) & $0(0 \%)$ & $3(10.3 \%)$ & 0.08 \\
\hline \multicolumn{4}{|l|}{ Desquamation severity } \\
\hline Lesions covering $<50 \%$ BSA with or without associated symptoms, $\mathrm{n}(\%)$ & $15(51.7 \%)$ & $16(55.2 \%)$ & 0.76 \\
\hline Lesions covering $\geq 50 \%$ BSA with or without associated symptoms, $\mathrm{n}(\%)$ & $1(3.4 \%)$ & $4(13.8 \%)$ & 0.16 \\
\hline \multicolumn{4}{|l|}{ Number of skin reactions } \\
\hline 1 type, n (\%) & $1(3.4 \%)$ & $4(10.3 \%)$ & 0.30 \\
\hline 2 types, n (\%) & $6(20.7 \%)$ & $7(24.1 \%)$ & 0.76 \\
\hline$\geq 3$ types, $\mathrm{n}(\%)$ & $22(75.9 \%)$ & $19(65.5 \%)$ & 0.34 \\
\hline
\end{tabular}

$p$-value $<0.05$ indicates statistical significance; Responders were defined as patients with stable disease and partial response. Non-responders were defined as patients with progressive disease. 
antibiotics (doxycycline and dicloxacillin) were used in patients with grade 3 severity. Low-dose systemic corticosteroids with a duration of less than 1 week were commenced in a few patients to rapidly reduce pain and skin inflammation. Moisturizers were recommended for use in all patients with xerosis and painful fissures on fingertips. Paronychia was treated with carbol-fuchsin in order to dry the wound and to prevent superinfection. There were no statistically significant differences in type, severity, or number of cutaneous reactions between responders and non-responders (Table 3 ).

\section{Discussion}

The EGFR family is a group of receptor tyrosine kinases that consists of four members: ErbB1, ErbB2, ErbB3, and ErbB4. In normal human skin, ErbB1 is expressed in the basal layer of the epidermis, the outer root sheath of the hair follicle, and the sebaceous and eccrine epitheliums. ${ }^{3,11}$ ErbB1 was also found to express in various types of cancers. ${ }^{3,11}$ Cetuximab, erlotinib, and gefitinib target ErbB1, and these drugs are increasingly used in patients with metastatic cancer. ${ }^{11}$ Due to their mechanisms of action, anti-EGFR therapies can cause both tumor suppression and cutaneous toxicities.

A retrospective study in Asians by Chanprapaph, et al. reported that $58.6 \%$ of patients taking erlotinib or gefitinib had skin reactions. ${ }^{7}$ Our study found a higher rate of skin reactions (96.7\%) from cetuximab, erlotinib, and gefitinib. Interestingly, both our study and the study by Chanprapaph, et al. were in Thai population. This difference may be due to one or more of the following factors: (i) the current study was a prospective study, (ii) skin examinations were performed by dermatologists, and (iii) skin reactions due to cetuximab were also investigated in our study. The incidence of xerosis due to EGFR inhibitor treatment was reported to vary from $4 \%$ to $100 \%{ }^{1,7,12-14}$ This wide variation in incidence may be due to dissimilarities in xerosis definition, climate, and duration of drug administration among studies. However and similar to a previous study, we found xerosis to be the most common reaction among Thai patients. ${ }^{7}$ Cetuximab was more likely to cause xerosis than erlotinib. ${ }^{9}$

Time duration to the development of each cutaneous reaction in Asian patients was comparable to the time durations found in Caucasian patients. ${ }^{1,714}$ In this study, acne/ papulopustular reaction, the most common reaction found in some other studies, developed between 1 and 3 weeks after the start of treatment. Paronychia and fissuring typically developed from 4 to 8 weeks after the start of treatment. ${ }^{14,18}$ The pathogenesis of cutaneous reactions is not well understood. Disruption in normal EGFR functions, which include maintaining and controlling skin integrity and inflammation, is believed to be the main pathogenic factor., ${ }^{9,15,16}$ For acne-like lesions, histopathology results showed 2 major patterns of reaction without sebaceous gland involvement, as follows: (i) suppurative folliculitis with associated rupture of epithelial lining; and, (ii) inflammatory cell infiltrate surrounding follicular infundibula, especially in the upper portion of the hair follicle. ${ }^{9,14,16}$ Similar to our study, bacterial cultures from pustular lesions revealed sterile pustule or Staphylococcus aureus overgrowth. ${ }^{14-17}$ Due to inconclusive pathogenesis and superimposed bacterial infection, corticosteroids, antibiotics, retinoids, and immunomodulatory agents have been used empirically to treat acne-like reaction with varying response. . $^{14,16,18}$

A randomized phase III study evaluated the effect of prophylactic treatment with minocycline for erlotinib-induced skin rash in 150 NSCLC patients. ${ }^{19}$ They found that prophylactic treatment did not decrease the incidence of skin reaction. Moreover, skin reaction was not self-limiting. Nevertheless, prophylactic treatment was able to reduce the severity of skin reaction, improve patient compliance, and did not interfere with the efficacy of erlotinib. Two meta-analyses involving 6,798 NSCLC patients treated with tyrosine kinase inhibitors (erlotinib and gefitinib) and 1,961 solid tumor patients treated with cetuximab reported that cutaneous reactions were found to be associated with better survival outcomes. ${ }^{8,20}$ Chanprapaph, et al. studied 99 Thai patients, which is the same ethnicity as the population in this study, and found that skin rashes were significantly associated with tumor response. ${ }^{7}$ We believe that our study was not able to demonstrate this same significant association due to the comparatively small size of our study population.

In conclusion, almost all patients in this study (96.7\%) developed cutaneous toxicities of EGFR inhibitors. Xerosis, acne, and skin desquamation were common cutaneous reactions. Most reactions were mild and were well tolerated with supportive treatment. Although current evidence suggests that cutaneous reactions seem to associate with tumor response, our study was not able to demonstrate this association due to limited number of patients.

\section{Acknowledgements}

The authors gratefully acknowledge the patients that agreed to participate in this study and Dr. Saowalak Hunnangkul for assistance with statistical analysis.

\section{Conflict of interest declaration}

The authors hereby declare no personal or professional conflicts of interest regarding any aspect of this study.

\section{Funding disclosure}

This was an unfunded study.

\section{References}

1. Roe E, Muret MPG, Marcuello E, Capdevila J, Pallares C, Alomar A. Description and management of cutaneous side effects during cetuximab or erlotinib treatments: a prospective study of 30 patients. J Am Acad Dermatol. 2006:55;429-37.

2. Kimyai-Asadi A, Jih MH. Follicular toxic effects of chimeric anti-epidermal growth factor receptor antibody cetuximab used to treat human solid tumors. Arch Dermatol. 2002;138:129-31.

3. Mahipal A, Kothari N, Gupta S. Epidermal Growth Factor Receptor Inhibitors: Coming of Age. Cancer Control. 2014;21:74-9.

4. Lacouture ME, Melosky BL. Cutaneous reactions to anticancer agents targeting the epidermal growth factor receptor: a dermatology-oncology perspective. Skin Therapy Lett. 2007;12:1-5.

5. Morse L, Calarese P. EGFR-targeted therapy and related skin toxicity. Semin Oncol Nurs. 2006;22:152-62.

6. Salf MW, Kim R. Incidence and management of cutaneous toxicities associated with cetuximab. Expert Opin Drug Saf. 2007;6:175-82. 
7. Chanprapaph K, Pongcharoen P, Vachiramon V. Cutaneous adverse events of epidermal growth factor receptor inhibitors: A retrospective review of 99 cases. Indian J Dermatol Venereol Leprol. 2015;81:547.

8. Liu HB, Wu Y, Lv TF, Yao YW, Xiao YY, Yuan DM, et al. Skin rash could predict the response to EGFR tyrosine kinase inhibitor and the prognosis for patients with non-small cell lung cancer: a systematic review and meta-analysis. PLoS One. 2013;8:e55128.

9. Curry JL, Torres-Cabala CA, Kim KB, Tetzlaff MT, Duvic M, Tsai KY, et al. Dermatologic toxicities to targeted cancer therapy: shared clinical and histologic adverse skin reactions. Int J Dermatol. 2014;53:376-84.

10. Eisenhauer EA, Therasse P, Bogaerts J, Schwartz LH, Sagen D, Ford R, et al. New response evaluation criteria in solid tumours: Revised RECIST guideline (version 1.1). Eur J Cancer. 2009;45:228-47.

11. Nair P. Epidermal growth factor receptor family and its role in cancer progression. Curr Sci. 2005;88:890-8.

12. Nakagawa $\mathrm{K}$, Kudoh $\mathrm{S}$, Ohe $\mathrm{Y}$, Johkoh $\mathrm{T}$, Ando $\mathrm{M}$, Yamazaki N, et al. Postmarketing surveillance study of erlotinib in Japanese patients with non-small-cell lung cancer (NSCLC): an interim analysis of 3488 patients (POLARSTAR). J Thorac Oncol. 2012;7:1296-303.

13. Osio A, Mateus C, Soria JC, Massard C, Malka D, Boige V, et al. Cutaneous side-effects in patients on long-term treatment with epidermal growth factor receptor inhibitors. Br J Dermatol. 2009;161:515-21.

14. Agero AL, Dusza SW, Benvenuto-Andrade C, Busam KJ, Myskowski P, Halpern AC. Dermatologic side effects associated with the epidermal growth factor receptor inhibitors. J Am Acad Dermatol. 2006;55:657-70.
15. Baselga J, Rischin D, Ranson M, Calvert H, Raymond E, Kieback DG, et al. Phase I safety, pharmacokinetic, and pharmacodynamic trial of ZD1839, a selective oral epidermal growth factor receptor tyrosine kinase inhibitor, in patients with five selected solid tumor types. J Clin Oncol. 2002;20: 4292-302.

16. Van Doorn R, Kirtschig G, Scheffer E, Stoof TJ, Giaccone G. Follicular and epidermal alterations in patients treated with ZD1839 (Iressa), an inhibitor of the epidermal growth factor receptor. Br J Dermatol. 2002;147:598-601.

17. Amitay-Laish I, David M, Stemmer SM. Staphylococcus coagulase-positive skin inflammation associated with epidermal growth factor receptor -targeted therapy: an early and a late phase of papulopustular eruptions. Oncologist. 2010;15:1002-8.

18. Hu JC, Sadeghi P, Pinter-Brown LC, Yashar S, Chiu MW. Cutaneous side effects of epidermal growth factor receptor inhibitors: clinical presentation, pathogenesis, and management. J Am Acad Dermatol. 2007;56:317-26.

19. Melosky B, Anderson H, Burkes RL, Chu Q, Ho V, Ho C. et al. Pan Canadian rash trial: a randomized phase III trial evaluating the impact of a prophylactic skin treatment regimen on epidermal growth factor receptor-tyrosine kinase inhibitor-induced skin toxicities in patients with metastatic lung cancer. J Clin Oncol. 2016;34:810-5.

20. Abdel-Rahman O, Fouad M. Correlation of cetuximab-induced skin rash and outcomes of solid tumor patients treated with cetuximab: a systematic review and meta-analysis. Crit Rev Oncol Hematol. 2015;93:127-35. 